Case Report

\title{
Targeted Next-Generation Resequencing of F5 Gene Identifies Novel Multiple Variants Pattern in Severe Hereditary Factor V Deficiency
}

\author{
Piotr K. Janicki, ${ }^{1}$ Sonia Vaida, ${ }^{1}$ and Hamid A. B. AL-Mondhiry ${ }^{2}$ \\ ${ }^{1}$ Laboratory of Perioperative Genomics, Department of Anesthesiology, Penn State University College of Medicine, \\ MS Hershey Medical Center, H187, 500 University Dr, Hershey, PA 17033, USA \\ ${ }^{2}$ Division of Hematology-Oncology, Penn State University College of Medicine, MS Hershey Medical Center, Hershey, PA 17033, USA
}

Correspondence should be addressed to Piotr K. Janicki; pjanicki@hmc.psu.edu

Received 29 January 2013; Accepted 19 February 2013

Academic Editors: S.-C. Chae, P. D. Cotter, A. Sazci, and C. Yapijakis

Copyright (C) 2013 Piotr K. Janicki et al. This is an open access article distributed under the Creative Commons Attribution License, which permits unrestricted use, distribution, and reproduction in any medium, provided the original work is properly cited.

\begin{abstract}
The present study investigated the genetic defects underlying severe Factor V deficiency in a 26-year-old Columbian (South America) female and her immediate family (both parents and newborn child) by next generation sequencing (NGS) of the entire F5 gene locus. Five mutations in the coding sequence of $F 5$, including three missense single-nucleotide variants (R2102H, R513K, D107H) and two synonymous variants (A135A, S184S), were identified and confirmed by the Sanger sequencing in the investigated proband (homozygote for all detected mutations), her parents, and her newborn child (all heterozygotic carriers for identified mutations). Each of the three missense variants was previously associated with separate phenotypes, including Factor V deficiency (R2102H), thrombosis (R513K) and frequent miscarriages (D107H). In addition, at least 75 additional single-nucleotide variants (including six novels) were identified in untranslated region of $F 5$.
\end{abstract}

\section{Introduction}

Coagulation Factor V is a large $330-\mathrm{kD}$ glycoprotein which consists of 2224 amino acid residues including a 28 -residue leader peptide, which is structurally and functionally homologous to coagulation Factor VIII $[1,2]$. The human Factor V gene (official name F5) maps to chromosome 1q23 and contains 25 exons (8). Factor $\mathrm{V}$ deficiency is a rare autosomal recessive disorder (incidence $<1$ in 1 million), characterized by low levels of antigen and activity [3]. At present, more than one hundred deficiency-causing mutations in the $F 5$ locus have been described, and although most of them are private, a few are common, being found in several individuals of both European, Middle-Eastern and Asiatic descents. In the present study, we used for the first time DNA next-generation sequence (NGS) analysis to detect mutation pattern in the entire F5 locus of a 26-year-old Hispanic parturient with severe Factor $\mathrm{V}$ deficiency, as well as in her asymptomatic parents and newborn baby. The relationship between combinations of mutations and clinical phenotypes was evaluated.

\section{Case Presentation}

The study protocol was approved by IRB at PSU Hershey Medical College. It was performed in adherence to the tenets of the declaration of Helsinki. Written informed consent was obtained from all participants. The investigated patient was 26-year-old Hispanic (born in Columbia, South America) parturient (G4P1) with several bleeding episodes before and during present pregnancy, multiple fresh frozen plasma (FFP) transfusions, and a history of three miscarriages in the past. The patient was previously diagnosed clinically to have severe Factor $\mathrm{V}$ deficiency on the basis of several previous bleeding episodes and laboratory studies demonstrating coagulopathy with moderate to severe decrease in Factor V activity. The remaining past medical history was unremarkable. The family history revealed that both biological parents had no history of bleeding or other coagulation symptoms and had reported normal Factor V activity. In addition, she has two siblings, apparently without any clinical signs of coagulation disorders. In the course of the current pregnancy the patient delivered 
the healthy male newborn, who has not displayed, at the time of this analysis, any signs of coagulation abnormalities, besides decreased (36\%) level of Factor V. For the purpose of this investigation, we collected the samples of blood (proband and newborn son) and saliva (both biological parents and proband's husband) for DNA analysis (Figure 1).

\section{Mutation Analysis}

Genomic DNA (gDNA) was extracted from venous EDTAwhole blood sample (proband) or cord blood (newborn child) employing membrane ultrafiltration method (FujiFilm Life Sciences distributed by Autogene, Holliston, MA, USA), according to the manufacturer recommendations. The saliva samples were collected from both parents and proband/s husband into Oragene container (DNA Genotek, Canada) and extracted according to the manufacturer recommendations. Subsequently two gDNA samples from the proband were submitted to Otogenetics Corporation (Norcross, GA, USA) for target capture and sequencing. Briefly, gDNA was subjected to agarose gel and optical density ratio tests to confirm the purity and concentration prior to Covaris (Covaris, Inc., Woburn, MA, USA) fragmentation. Fragmented gDNAs were tested for size distribution and concentration using an Agilent Bioanalyzer 2100 (Agilent Technologies, Santa Clara, CA, USA) and Nanodrop (Thermo Fisher Scientific, Wilmington DE, USA). Illumina libraries were made from qualified fragmented gDNA using NEBNext reagents (New England Biolabs, Ipswich, MA, USA) and the resulting libraries were subjected to exome enrichment using custom probes targeting $75 \mathrm{~kb}$ target on chromosome 1 (169, 481, 192-169, 555, 469 by GRCh 37, Hg19). The resultant libraries were tested for enrichment by qPCR and for size distribution and concentration by an Agilent Bioanalyzer 2100. The samples were then sequenced on an Illumina HiSeq2000 (Illumina, San Diego, CA, USA) which generated pairedend reads of 90 or 100 nucleotides. Data was analyzed for data quality, exome coverage, and exome-wide SNP/InDel using the platform provided by DNAnexus (DNAnexus, Inc, Mountain View, CA, USA). The detected polymorphisms in the coding sequence of the F5 locus were subsequently verified using classical Sanger sequencing using exon primers described by van Wijk et al. [4]. This analysis was performed by direct DNA sequencing using ABI Prism 3100 genetic analyzer (Applied Biosystems, Foster City, CA, USA).

By NGS, we generated about 549 million bases of sequence as pair-end 90 or 100 nucleotide reads, $86 \%$ of which was able to align to human reference sequence. A total of $23 \%$ of these sequences mapped to the targeted region corresponding to $75 \mathrm{~kb}$ sequence of the F5 locus (NM_0001304.4), with 540 -fold mean coverage (Table 1). At this depth of coverage, more than $95 \%$ of the target bases were covered to pass quality control filtering based on the PHRED score threshold of calling variants (PHRED > 30). Eighty high-confidence variants were annotated in the target region (3 nonsynonymous single-nucleotide variants and 2 synonymous singlenucleotide variants in the coding region of the target (Table 2), as well as 75 single-nucleotide variants in the

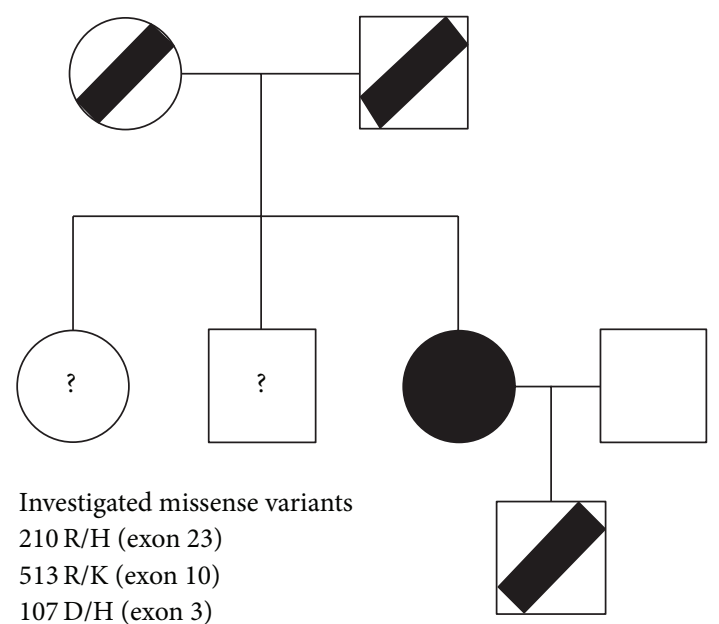

FIGURE 1: Segregation of $F 5$ missense variants $2102 \mathrm{R} / \mathrm{H}$ (exon 23), $513 \mathrm{R} / \mathrm{K}$ (exon 10) and $107 \mathrm{D} / \mathrm{H}$ (exon 3). Half-filled symbols indicate heterozygotes for investigated mutatons. Completely filled symbols indicate homozygous for all investigated mutations (i.e., proband). Open symbols indicate no carrier of investigated mutations. Question marks indicate that genotyping has not been performed.

noncoding sequence of the target (Table 3). The missense variants were located in the exon 3 (D107H), 10 (R513K), and $23(\mathrm{R} 2102 \mathrm{H})$ of the F5 locus (Figure 1), and the investigated patient was a homozygous carrier for all these variants. These variants were additionally confirmed by the Sanger sequencing and showed perfect match in two duplicate samples. The additional verification of the DNA samples from the proband's newborn son and our patient's biological parents performed using the Sanger sequencing revealed that all of them were heterozygous carriers for all 3 missense variants (Figure 2). No presence of these variants was shown in the husband of the patient (i.e., biological father of the newborn child).

\section{Discussion}

The current study presents several novel findings: (i) it employs NGS approach for molecular diagnosis of FV deficiency, including sequencing of both translated and untranslated regions of F5 locus; (ii) it diagnoses the inherited FV deficiency in the S. American Caucasian family of Hispanic ethnicity; and (iii) it depicts detailed genetic evidence for multiple, coinherited mutations in the F5 locus which may be responsible for opposite phenotype characteristics (i.e., increasing and decreasing thrombosis process).

The results of the current study indicate that the investigated proband was a homozygotic carrier of three separate missense mutations in the coding sequence of F5. Surprisingly, however, only one of the 3 detected missense mutations $(\mathrm{R} 2102 \mathrm{H})$ was described previously (and called at that time, $\mathrm{R} 2074 \mathrm{H}$, based on different amino acid number order) by Schijver et al. (2002) in the context of the deficiency of 
1 mfpgcprLwv LvvLgtswvg wgsqgteaaq lrqfyvaaqg iswsyrpept nsslnlsvts

61 fkkivyreye pyfkkekpqs tisgllgptl yaevgdiikv hfknkad->hkpl sihpqgirys

$121 \mathrm{klsegasyld}$ htfpa->aekmdd avapgreyty ewsisedsgp thddppclth iyyshenlie

181 dfns->sgligpl lickkgtlte ggtqktfdkq ivllfavfde skswsqsssl mytvngyvng

241 tmpditvcah dhiswhllgm ssgpelfsih fngqvleqnh hkvsaitlvs atsttanmtv

301 gpegkwiiss ltpkhlqagm qayidikncp kktrnlkkit reqrrhmkrw eyfiaaeevi

361 wdyapvipan mdkkyrsqhl dnfsnqigkh ykkvmytqye desftkhtvn pnmkedgilg

421 piiraqvrdt lkivfknmas rpysiyphgv tfspyedevn ssftsgrnnt miravqpget

481 ytykwnilef deptendaqc ltrpyysdvd im $r->k d i a s g l i$ glllicksrs ldrrgiqraa

541 dieqqavfav fdenkswyle dninkfcenp devkrddpkf yesnimstin gyvpesittl

601 gfcfddtvqw hfcsvgtqne iltihftghs fiygkrhedt ltlfpmrges vtvtmdnvgt

661 wmltsmnssp rskklrlkfr dvkcipddde dsyeifeppe stvmatrkmh drlepedees

721 dadydyqnrl aaalgirsfr nsslnqeeee fnltalalen gtefvssntd iivgsnyssp

781 sniskftvnn laepqkapsh qqattagspl rhligknsvl nsstaehssp ysedpiedpl

841 qpdvtgirll slgagefksq ehakhkgpkv erdqaakhrf swmkllahkv grhlsqdtgs

901 psgmrpwedl psqdtgspsr mrpwkdppsd llllkqsnss kilvgrwhla sekgsyeiiq

961 dtdedtavnn wlispqnasr awgestplan kpgkqsghpk fprvrhkslq vrqdggksrl

1021 kksqfliktr kkkkekhthh aplsprtfhp lrseayntfs errlkhslvl hksnetslpt

1081 dlnqtlpsmd fgwiaslpdh nqnssndtgq ascppglyqt vppeehyqtf piqdpdqmhs

1141 tsdpshrsss pelsemleyd rshksfptdi sqmspssehe vwqtvispdl sqvtlspels

1201 qtnlspdlsh ttlspeliqr nlspalgqmp ispdlshttl spdlshttls ldlsqtnlsp

1261 elsqtnlspa lgqmplspdl shttlsldfs qtnlspelsh mtlspelsqt nlspalgqmp

1321 ispdlshttl sldfsqtnls pelsqtnlsp algqmplspd pshttlsldl sqtnlspels

1381 qtnlspdlse mplfadlsqi pltpdldqmt lspdlgetdl spnfgqmsls pdlsqvtlsp

1441 disdttllpd lsqispppdl dqifypsess qslllqefne sfpypdlgqm pspssptlnd

1501 tflskefnpl vivglskdgt dyieiipkee vqsseddyae idyvpyddpy ktdvrtnins

1561 srdpdniaaw ylrsnngnrr nyyiaaeeis wdysefvqre tdiedsddip edttykkvvf

1621 rkyldstftk rdprgeyeeh lgilgpiira evddviqvrf knlasrpysl hahglsyeks

1681 segktyedds pewfkednav qpnssytyvw hatersgpes pgsacraway ysavnpekdi

1741 hsgligplli cqkgilhkds nmpmdmrefv llfmtfdekk swyyekksrs swrltssemk

1801 kshefhaing miyslpglkm yeqewvrlhl lniggsqdih vvhfhgqtll engnkqhqlg

1861 vwpllpgsfk tlemkaskpg wwllntevge nqragmqtpf limdrdcrmp mglstgiisd

1921 sqikaseflg yweprlarln nggsynawsv eklaaefask pwiqvdmqke viitgiqtqg

1981 akhylkscyt tefyvayssn qinwqifkgn strnvmyfng nsdastiken qfdppivary

2041 irisptrayn rptlrlelqg cevngcstpl gmengkienk qitassfkks wwgdywepfr

2101 ar->hlnaqgrvn awqakannnk qwleidllki kkitaiitqg ckslssemyv ksytihyseq

2161 gvewkpyrlk ssmvdkifeg ntntkghvkn ffnppiisrf irvipktwnq sialrlelfg

2221 cdiy

Figure 2: Amino acid sequence of human factor V deducted from the F5 DNA sequence of proband. Red fonts indicate missense mutations, and blue fonts indicate synonymous mutations. The sequence of first 28 amino acid fragments is given in italics, and the consecutive 25 exons are either underlined or not underlined (pair wise).

TABLE 1: Summary of coverage for analyzed samples by next generation sequencing method.

\begin{tabular}{lccccc}
\hline Sample & Average target coverage & Total bases & Total reads & Mapped reads & Bottleneck score \\
\hline Original proband DNA sample & 547 & $583,280,200$ & $5,832,802$ & $4,964,887$ & 31.81 \\
Duplicate & 534 & $549,426,400$ & $5,494,264$ & $4,720,781$ & 32.81 \\
\hline
\end{tabular}

the Factor $\mathrm{V}$ [5]. This substitution results in the replacement of an arginine $(\mathrm{R})$ by a histidine $(\mathrm{H})$ in amino acid position 2102 located in the $\mathrm{C} 2$-domain of Factor $\mathrm{V}$ and several lines of evidence reported previously support the notion that this sequence variant is causative for Factor $\mathrm{V}$ deficiency phenotype. Interestingly the remaining two detected missense mutations were reported to be associated mostly with either thrombosis [6-8] or increased risk of preterm delivery $(\mathrm{D} 107 \mathrm{H})$ [9]. It is noteworthy that two missense point mutations which were detected in the current proband were previously described in different populations (R2102H in Tunisian population and $\mathrm{R} 513 \mathrm{~K}$ in Thai, Chinese and Sub-Saharan populations). Although Factor $\mathrm{V}$ deficiency and its causative mutations were reported previously from European Caucasians, it is, to our knowledge, the first detailed description of causative mutation in F5 locus in a family from South America and/or Hispanic ethnicity.
In addition, we established that the investigated proband was a homozygous carrier for two synonymous singlenucleotide variants: A135A (rs6029) and S184S (rs6022) previously reported in the online SNP Medline database. There is no information about the potential phenotypic significance of these mutations. In addition to the previously described single-point mutations in the coding region of $F 5$ gene, the analysis of the NGS data from the proband established a presence of additional 75 polymorphisms in the untranslated sequence of the $F 5$ locus ( 3 in $5^{\prime}$-UTR part, 71 in the intronic part, and 1 in the $3^{\prime}$-UTR part of the gene) (Table 3 ). Six of these variants represent newly discovered variants, not presented previously in the SNP Medline database. The types of detected variants include single-nucleotide variants (4 insertions and 4 deletions), as well as 3 additional short indels. The phenotypic significance of these polymorphisms for the Factor $\mathrm{V}$ function remains unknown. 
TABLE 2: Mutations observed in coding sequences (CDS) of $F 5$ gene in the investigated proband and her blood relatives.

\begin{tabular}{|c|c|c|c|c|c|}
\hline Patient & Bleeding phenotype & $\begin{array}{c}\text { FV activity } \\
(\%)\end{array}$ & Variants in CDS of F5 gene & Mature FV protein variants & SNPs (ID) \\
\hline \multirow{7}{*}{$\begin{array}{l}\text { Female } \\
\text { proband }\end{array}$} & \multirow{7}{*}{$\begin{array}{l}\text { Epistaxis multiple } \\
\text { miscarriages }\end{array}$} & \multirow{7}{*}{$0-4 \%$} & Homozygote for nonsynonymous variants: & & \\
\hline & & & $169486641 \mathrm{G}>\mathrm{C}$ & 2102 R/H (exon 23) & No dbSNP ID \\
\hline & & & $169519112 \mathrm{C}>\mathrm{T}$ & 513 R/K (exon 10) & rs6020 \\
\hline & & & $169541513 \mathrm{C}>\mathrm{G}$ & 107 D/H (exon 3) & rs6019 \\
\hline & & & Homozygote for synonymous variants: & & \\
\hline & & & $169529973 \mathrm{C}>\mathrm{T}$ & $135 \mathrm{~A} / \mathrm{A}$ (exon 4) & rs6029 \\
\hline & & & $169529826 \mathrm{C}>\mathrm{A}$ & $184 \mathrm{~S} / \mathrm{S}($ exon 4$)$ & rs6022 \\
\hline \multirow{7}{*}{$\begin{array}{l}\text { Newborn } \\
\text { son of } \\
\text { proband }\end{array}$} & \multirow{7}{*}{ No bleeding } & \multirow{7}{*}{$36 \%$} & Heterozygote for nonsynonymous variants: & & \\
\hline & & & $169486641 \mathrm{G}>\mathrm{C}$ & 2102 R/H (exon 23) & No dbSNP ID \\
\hline & & & $169519112 \mathrm{C}>\mathrm{T}$ & $513 \mathrm{R} / \mathrm{K}$ (exon 10) & rs6020 \\
\hline & & & 169541513 C > G & $107 \mathrm{D} / \mathrm{H}($ exon 3$)$ & rs6019 \\
\hline & & & Heterozygote for synonymous variants: & & \\
\hline & & & $169529973 \mathrm{C}>\mathrm{T}$ & $135 \mathrm{~A} / \mathrm{A}($ exon 4$)$ & rs6029 \\
\hline & & & $169529826 \mathrm{C}>\mathrm{A}$ & 184 S/S (exon 4) & rs6022 \\
\hline \multirow{7}{*}{$\begin{array}{l}\text { Mother of } \\
\text { proband }\end{array}$} & \multirow{7}{*}{ No bleeding } & \multirow{7}{*}{$\begin{array}{l}\text { Reported } \\
\text { normal }\end{array}$} & Heterozygote for nonsynonymous variants: & & \\
\hline & & & $169486641 \mathrm{G}>\mathrm{C}$ & 2102 R/H (exon 23) & No dbSNP ID \\
\hline & & & $169519112 \mathrm{C}>\mathrm{T}$ & 513 R/K (exon 10) & rs6020 \\
\hline & & & $169541513 \mathrm{C}>\mathrm{G}$ & 107 D/H (exon 3) & rs6019 \\
\hline & & & Heterozygote for synonymous variants: & & \\
\hline & & & $169529973 \mathrm{C}>\mathrm{T}$ & $135 \mathrm{~A} / \mathrm{A}($ exon 4$)$ & rs6029 \\
\hline & & & $169529826 \mathrm{C}>\mathrm{A}$ & 184 S/S (exon 4) & rs6022 \\
\hline \multirow{7}{*}{$\begin{array}{l}\text { Father of } \\
\text { proband }\end{array}$} & \multirow{7}{*}{ No bleeding } & \multirow{7}{*}{$\begin{array}{l}\text { Reported } \\
\text { normal }\end{array}$} & Heterozygote for nonsynonymous variants: & & \\
\hline & & & $169486641 \mathrm{G}>\mathrm{C}$ & 2102 R/H (exon 23) & No dbSNP ID \\
\hline & & & $169519112 \mathrm{C}>\mathrm{T}$ & 513 R/K (exon 10) & rs6020 \\
\hline & & & 169541513 C > G & $107 \mathrm{D} / \mathrm{H}($ exon 3$)$ & rs6019 \\
\hline & & & Heterozygote for synonymous variants: & & \\
\hline & & & $169529973 \mathrm{C}>\mathrm{T}$ & 135 A/A (exon 4) & rs6029 \\
\hline & & & $169529826 \mathrm{C}>\mathrm{A}$ & $184 \mathrm{~S} / \mathrm{S}($ exon 4$)$ & rs6022 \\
\hline $\begin{array}{l}\text { Proband's } \\
\text { husband }\end{array}$ & No bleeding & $\begin{array}{l}\text { Reported } \\
\text { normal }\end{array}$ & No above CDS variants detected in F5 & - & - \\
\hline
\end{tabular}

The most recently (Oct 2012) accessed Human Genome Mutation database (www.hgmd.org) lists 145 missense mutations in the F5 locus associated with altered function of Factor V. From this list, 94 mutations represent single-point mutations, from which approximately 80 have been strongly associated with Factor $\mathrm{V}$ deficiency. In this respect, the present study does not add new mutations to this list but confirms the fact of previously described coinheritance of several separate mutations in the F5 locus $[10,11]$, and more importantly provides an example of co-inheritance of $F 5$ mutations with presumably opposite phenotypic coagulation characteristics. Similar situation (i.e., coinheritance of polymorphic variants from which one is associated with decreased Factor $\mathrm{V}$ activity and other with increased thrombosis) was described previously for much more frequent prothrombotic Leiden mutations, or more recently, promoter $-426 \mathrm{G} / \mathrm{A}$ polymorphism $[12,13]$. Our finding confirms that other prothrombotic mutations in the F5 gene locus may be independently inherited in one patient.

The present study exemplifies the use of NGS approach for detailed diagnosis of the specific clinical pathology and identification of causative mutations for rare genetic disorder. This approach has been recently advocated for both diagnosis and therapy [14]. The obtained genetic data for this cases were successfully used clinically for the peripartum anesthetic management of the previously described patient 
TABLE 3: Summary of observed variants in noncoding sequences of $F 5$ gene in investigated proband.

\begin{tabular}{|c|c|c|c|c|c|c|}
\hline Type of variant & Type of carrier & Position-R & Position-L & Reference base & Variant base & SNPs (ID) \\
\hline \multicolumn{7}{|c|}{ Variants in $5^{\prime}$-UTR } \\
\hline SNP & Hom & 169556050 & 169556051 & c & $\mathrm{t}$ & rs2269648 \\
\hline SNP & Hom & 169556152 & 169556153 & g & $\mathrm{t}$ & New variant \\
\hline INS & Het & 169556812 & 169556812 & & a & rs58897818 \\
\hline \multicolumn{7}{|c|}{ Intronic variants } \\
\hline SNP & Hom & 169486641 & 169486642 & g & c & rs9332666 \\
\hline SNP & Hom & 169490392 & 169490393 & g & c & rs2420370 \\
\hline SNP & Hom & 169491555 & 169491556 & g & $\mathrm{a}$ & rs2420371 \\
\hline SNP & Hom & 169496536 & 169496537 & g & c & New variant \\
\hline SNP & Hom & 169498056 & 169498057 & a & g & rs2420372 \\
\hline SNP & Hom & 169516507 & 169516508 & g & a & rs12046953 \\
\hline SNP & Hom & 169517159 & 169517160 & c & $\mathrm{t}$ & rs12026997 \\
\hline SNP & Hom & 169517385 & 169517386 & a & g & rs12044669 \\
\hline SNP & Hom & 169517904 & 169517905 & g & a & rs 2420374 \\
\hline SNP & Hom & 169518703 & 169518704 & $\mathrm{t}$ & c & rs58875232 \\
\hline DEL & Het & 169518885 & 169518886 & a & & rs55717622 \\
\hline SNP & Hom & 169519417 & 169519418 & g & a & rs7537742 \\
\hline SNP & Hom & 169519765 & 169519766 & a & g & rs13306331 \\
\hline SNP & Hom & 169520098 & 169520099 & a & g & rs10800456 \\
\hline SNP & Hom & 169521553 & 169521554 & g & $\mathrm{a}$ & rs2213868 \\
\hline SNP & Hom & 169521733 & 169521734 & c & $\mathrm{t}$ & rs9332582 \\
\hline SNP & Hom & 169523346 & 169523347 & a & g & rs7555832 \\
\hline SNP & Hom & 169523389 & 169523390 & $\mathrm{t}$ & c & rs11577059 \\
\hline SNP & Het & 169523395 & 169523396 & a & $\mathrm{t}$ & New variant \\
\hline SNP & Het & 169523396 & 169523397 & a & g & New variant \\
\hline DEL & Hom & 169523397 & 169523398 & $\mathrm{t}$ & & rs116132528 \\
\hline INS & Het & 169524860 & 169524860 & & $\mathrm{cac}$ & New variant \\
\hline SNP & Het & 169524865 & 169524866 & $\mathrm{a}$ & $\mathrm{g}$ & New variant \\
\hline SNP & Hom & 169525312 & 169525313 & c & $\mathrm{t}$ & rs9332579 \\
\hline INS & Het & 169525558 & 169525558 & & $\operatorname{ctctggc}$ & rs16684 \\
\hline SNP & Het & 169525680 & 169525681 & c & $\mathrm{a}$ & rs115199761 \\
\hline SNP & Hom & 169525766 & 169525767 & $\mathrm{t}$ & c & rs 2239853 \\
\hline INS & Hom & 169526266 & 169526266 & & a & rs9332577 \\
\hline SNP & Hom & 169526300 & 169526301 & c & $\mathrm{t}$ & rs4656688 \\
\hline SNP & Hom & 169526367 & 169526368 & a & g & rs4656689 \\
\hline SNP & Hom & 169526425 & 169526426 & g & $\mathrm{t}$ & rs4656188 \\
\hline SNP & Hom & 169526601 & 169526602 & g & c & rs1894697 \\
\hline SNP & Hom & 169526646 & 169526647 & a & g & rs1894698 \\
\hline SNP & Hom & 169526950 & 169526951 & c & g & rs1894699 \\
\hline SNP & Hom & 169527226 & 169527227 & $\mathrm{a}$ & g & rs1981491 \\
\hline DEL & Hom & 169527470 & 169527471 & $\mathrm{a}$ & & rs3835454 \\
\hline SNP & Hom & 169528075 & 169528076 & c & $\mathrm{g}$ & rs9332570 \\
\hline INS & Het & 169528255 & 169528255 & & aaa & rs58738850 \\
\hline SNP & Hom & 169528580 & 169528581 & c & $\mathrm{t}$ & rs6012 \\
\hline SNP & Hom & 169528722 & 169528723 & c & $\mathrm{t}$ & rs6427201 \\
\hline SNP & Hom & 169528830 & 169528831 & c & $\mathrm{t}$ & rs6427202 \\
\hline SNP & Hom & 169529031 & 169529032 & $\mathrm{a}$ & c & rs6427203 \\
\hline SNP & Hom & 169529132 & 169529133 & c & $\mathrm{t}$ & rs6699691 \\
\hline SNP & Hom & 169529138 & 169529139 & c & $\mathrm{t}$ & rs58931047 \\
\hline SNP & Hom & 169530070 & 169530071 & $\mathrm{a}$ & c & rs7545236 \\
\hline SNP & Hom & 169530077 & 169530078 & g & $\mathrm{t}$ & rs7523043 \\
\hline
\end{tabular}


TABLE 3: Continued.

\begin{tabular}{|c|c|c|c|c|c|c|}
\hline Type of variant & Type of carrier & Position-R & Position-L & Reference base & Variant base & SNPs (ID) \\
\hline SNP & Hom & 169530093 & 169530094 & $\mathrm{t}$ & $\mathrm{c}$ & rs7534848 \\
\hline SNP & Hom & 169530176 & 169530177 & c & g & rs7522982 \\
\hline INS & Hom & 169530532 & 169530532 & & $\mathrm{a}$ & rs5778621; rs77192101 \\
\hline SNP & Hom & 169530586 & 169530587 & $\mathrm{t}$ & c & rs1894701 \\
\hline SNP & Hom & 169531442 & 169531443 & $\mathrm{t}$ & c & rs7540556 \\
\hline SNP & Hom & 169531571 & 169531572 & $\mathrm{t}$ & c & rs 4656690 \\
\hline SNP & Hom & 169533266 & 169533267 & g & a & rs6678795 \\
\hline SNP & Hom & 169533744 & 169533745 & $\mathrm{t}$ & $\mathrm{a}$ & rs724509 \\
\hline SNP & Hom & 169534028 & 169534029 & $\mathrm{t}$ & c & rs724507 \\
\hline SNP & Hom & 169534966 & 169534967 & $\mathrm{t}$ & $\mathrm{a}$ & rs 2040443 \\
\hline SNP & Hom & 169535353 & 169535354 & c & $g$ & rs6685578 \\
\hline SNP & Hom & 169536167 & 169536168 & $\mathrm{t}$ & c & rs2213869 \\
\hline SNP & Hom & 169536650 & 169536651 & g & $\mathrm{a}$ & rs2187955 \\
\hline DEL & Het & 169536796 & 169536798 & $\mathrm{tt}$ & $\mathrm{t}$ & New variant \\
\hline SNP & Hom & 169537678 & 169537679 & $\mathrm{a}$ & g & rs6670678 \\
\hline SNP & Hom & 169538466 & 169538467 & g & $\mathrm{a}$ & rs9287095 \\
\hline SNP & Hom & 169538544 & 169538545 & c & $\mathrm{t}$ & rs2298908 \\
\hline SNP & Hom & 169538603 & 169538604 & $\mathrm{t}$ & g & rs2298906 \\
\hline SNP & Hom & 169539348 & 169539349 & $\mathrm{t}$ & g & rs6663533 \\
\hline SNP & Hom & 169543263 & 169543264 & c & a & rs10800457 \\
\hline SNP & Hom & 169545413 & 169545414 & a & g & rs6677374 \\
\hline SNP & Hom & 169549775 & 169549776 & $\mathrm{t}$ & c & rs10753787 \\
\hline INS & Het & 169551560 & 169551560 & & $\mathrm{a}$ & rs56901113 \\
\hline SNP & Hom & 169554058 & 169554059 & $\mathrm{c}$ & g & rs3753305 \\
\hline \multicolumn{7}{|c|}{ Variants in $3^{\prime}$-UTR } \\
\hline SNP & Hom & 169479974 & 169479975 & $\mathrm{c}$ & $\mathrm{t}$ & rs970740 \\
\hline
\end{tabular}

Hom: homozygote, Het: heterozygote, SNP: single-nucleotide polymorphism, Del: deletion, and Ins: insertion

[15]. Comprehensive genetic analysis through NGS based approaches will increasingly be helpful in establishing the diagnosis of Factor $\mathrm{V}$ deficiency (or other genetic coagulation disorders) and thereby improve patient management.

\section{Conflict of Interests}

The authors declare that there is no conflict of interests.

\section{References}

[1] R. J. Jenny, D. D. Pittman, and J. J. Toole, "Complete cDNA and derived amino acid sequence of human Factor V," Proceedings of the National Academy of Sciences of the United States of America, vol. 84, no. 14, pp. 4846-4850, 1987.

[2] J. N. Huang and M. A. Koerper, "Factor V deficiency: a concise review," Haemophilia, vol. 14, no. 6, pp. 1164-1169, 2008.

[3] P. A. Owren, "Parahaemophilia-haemorrhagic diathesis due to absence of a previously unknown clotting factor," The Lancet, vol. 1, no. 6449, pp. 446-448, 1947.

[4] R. van Wijk, K. Nieuwenhuis, M. van den Berg et al., "Five novel mutations in the gene for human blood coagulation Factor $\mathrm{V}$ associated with type I Factor V deficiency," Blood, vol. 98, no. 2, pp. 358-367, 2001.
[5] I. Schrijver, R. Houissa-Kastally, C. D. Jones, K. C. Garcia, and J. L. Zehnder, "Novel Factor V C2-domain mutation (R2074H) in two families with Factor V deficiency and bleeding," Thrombosis and Haemostasis, vol. 87, no. 2, pp. 294-299, 2002.

[6] D. Helley, C. Besmond, R. Ducrocq et al., "Polymorphism in exon 10 of the human coagulation Factor $\mathrm{V}$ gene in a population at risk for sickle cell disease," Human Genetics, vol. 100, no. 2, pp. 245-248, 1997.

[7] M. Hiyoshi, P. Arnutti, W. Prayoonwiwat et al., "A polymorphism nt $1628 \mathrm{G} \rightarrow \mathrm{A}(\mathrm{R} 485 \mathrm{~K})$ in exon 10 of the coagulation Factor $\mathrm{V}$ gene may be a risk factor for thrombosis in the indigenous Thai population," Thrombosis and Haemostasis, vol. 80, no. 4, pp. 705-706, 1998.

[8] W. Le, J. D. Yu, L. Lu et al., "Association of the R485K polymorphism of the Factor $\mathrm{V}$ gene with poor response to activated protein $\mathrm{C}$ and increased risk of coronary artery disease in the Chinese population," Clinical Genetics, vol. 57, no. 4, pp. 296303, 2000.

[9] Y. Yu, H. J. Tsai, X. Liu et al., “The joint association between F5 gene polymorphisms and maternal smoking during pregnancy on preterm delivery," Human Genetics, vol. 124, no. 6, pp. 659$668,2009$.

[10] L. Cao, Z. Wang, H. Li et al., "Gene analysis and prenatal diagnosis for two families of congenital factor V deficiency," Haemophilia, vol. 17, no. 1, pp. 65-69, 2011. 
[11] V. Bafunno, G. Favuzzi, T. Fierro et al., "Coinheritance of three novel FV gene mutations in a patient with a severe FV deficiency," Haemophilia, vol. 18, no. 2, pp. e51-e53, 2012.

[12] P. Simioni, E. Castoldi, B. Lunghi, D. Tormene, J. Rosing, and F. Bernardi, "An underestimated combination of opposites resulting in enhanced thrombotic tendency," Blood, vol. 106, no. 7, pp. 2363-2365, 2005.

[13] A. Ozturk and N. Akar, "The effect of FV-426 G/A gene variation on the occurrence of thrombosis," Clinical \& Applied Thrombosis. In press.

[14] A. N. Desai and A. Jere, "Next-generation sequencing: ready for the clinics?" Clinical Genetics, vol. 81, no. 6, pp. 503-510, 2012.

[15] S. Vaida, H. Al-Mondhiry, D. Bezinover et al., "Peripartum anesthetic management of a parturitient with inherited factor $\mathrm{V}$ deficiency," Anesthesia \& Analgesia. In press. 


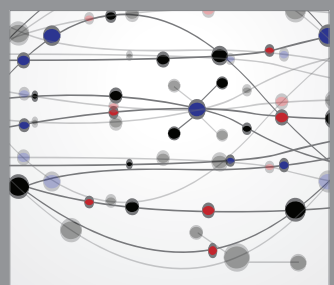

The Scientific World Journal
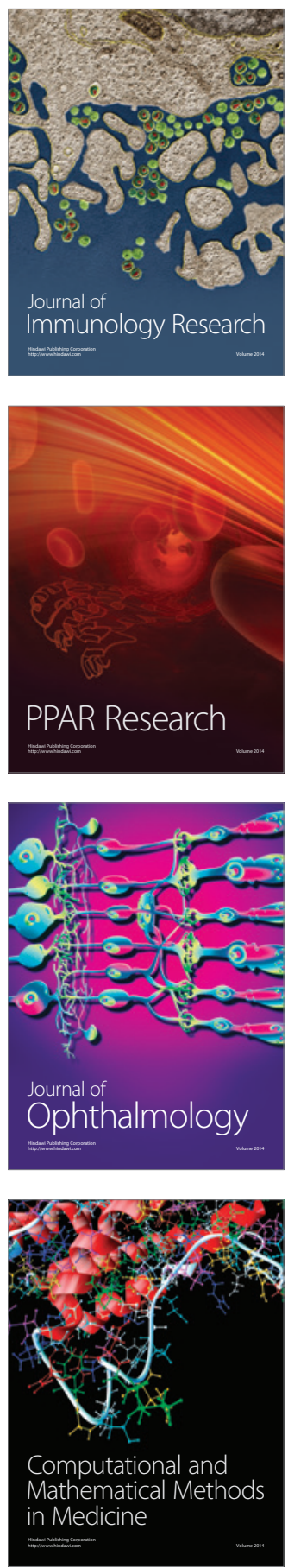

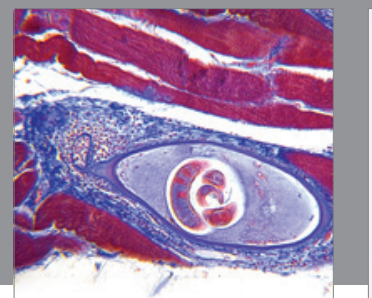

Gastroenterology

Research and Practice
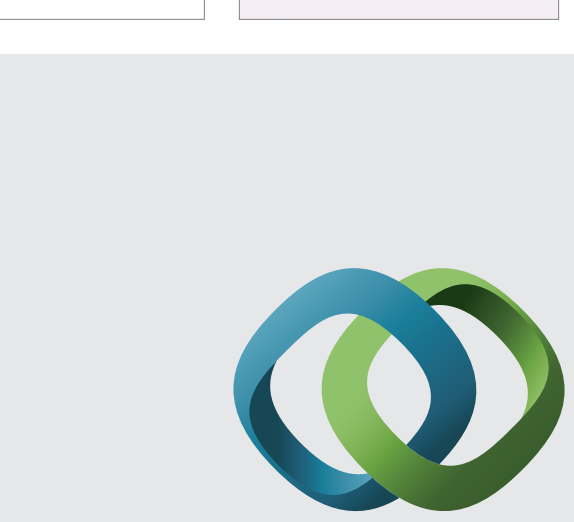

\section{Hindawi}

Submit your manuscripts at

http://www.hindawi.com
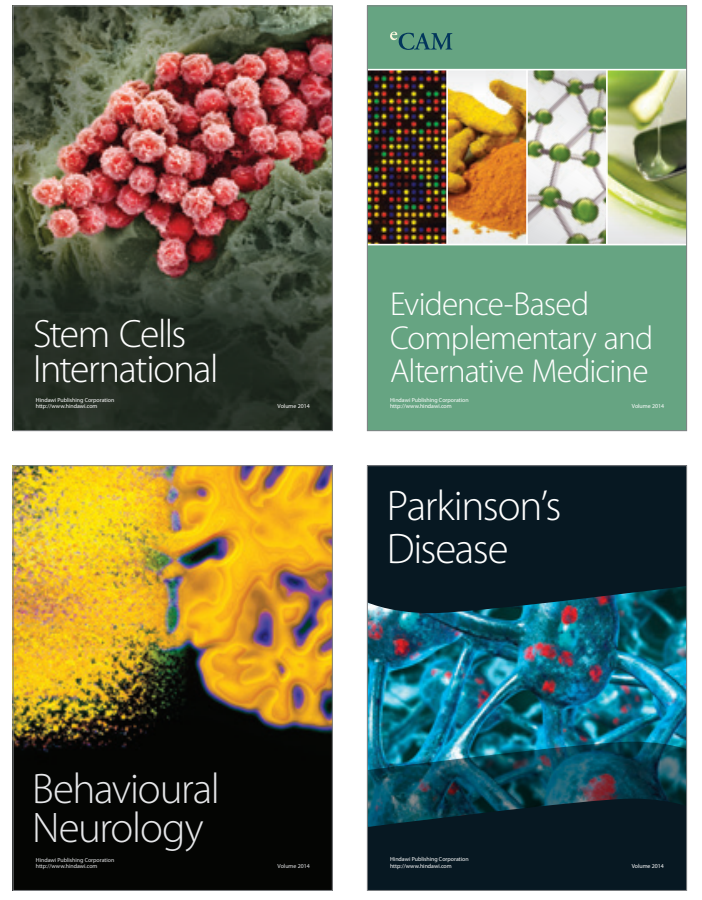
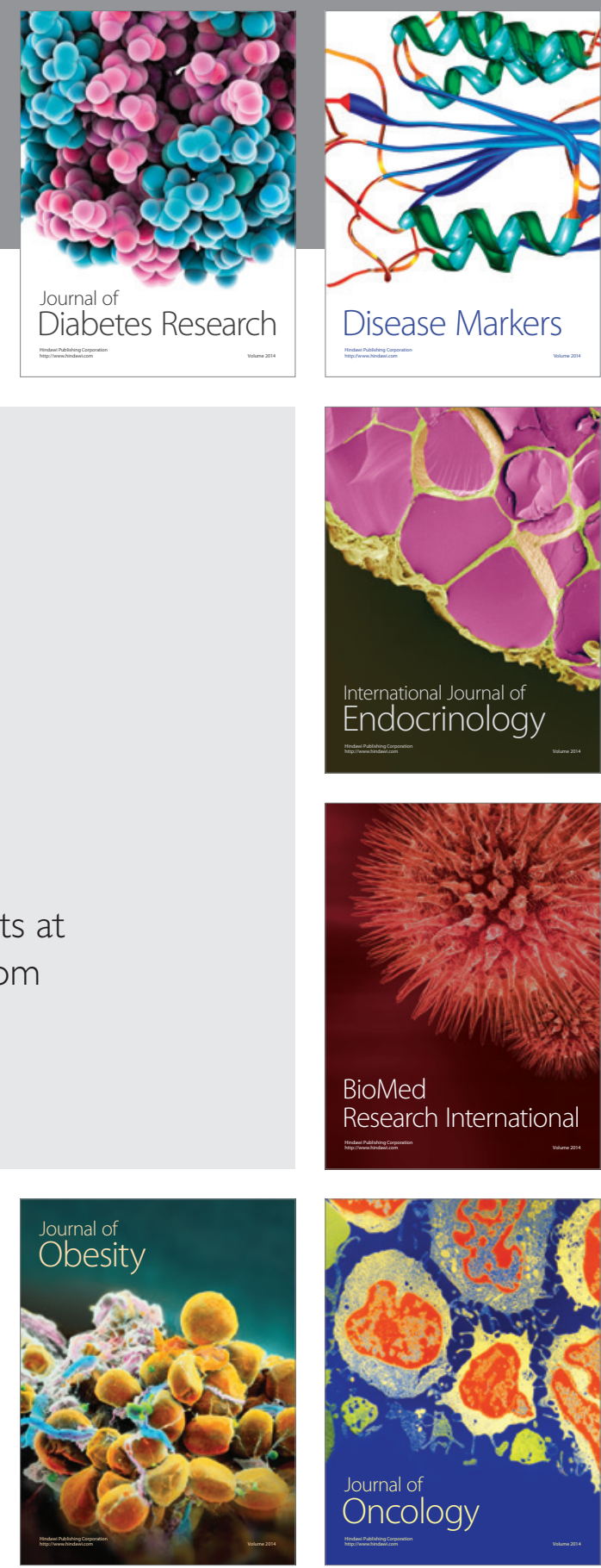

Disease Markers
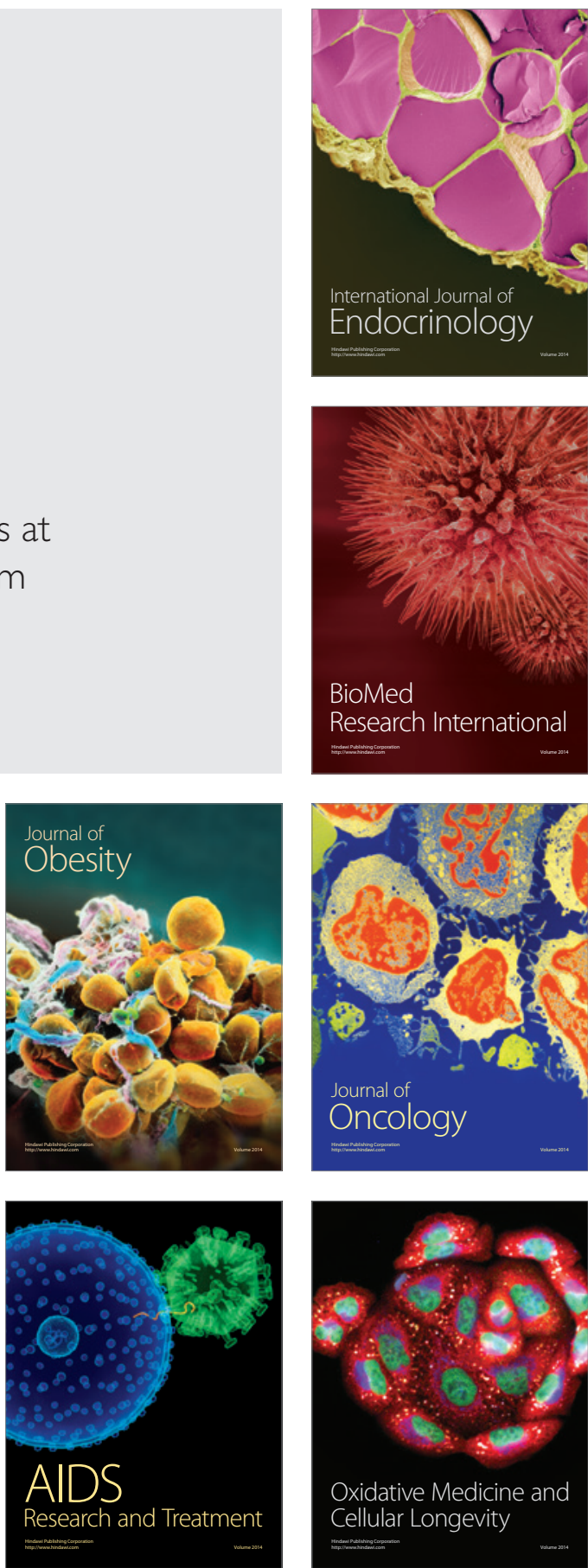